\title{
Overview Of LTE-A Technology
}

\author{
Sravanthi Kanchi, Shubhrika Sandilya, Deesha Bhosale, Adwait Pitkar, Mayur Gondhalekar
}

\begin{abstract}
Along with the rapid development in cellular technology, there has also been a significant increase in its user demands. Ever since LTE technology has been established in 2009, the work on its enhancements and requirements had begun and these have been fulfilled successfully by LTEAdvanced. The 3GPP Release 10 or LTE-A has proven to be one of the fastest developing mobile technologies in the world. Further improvements of LTE-A is still on the go and its future releases have enhanced features of MIMO, Carrier Aggregation, co-ordinated multipoint transmission (CoMP), high data rates and speed in order to provide the best user experience as well as proving to be a complete 4G network. In this paper, an overview on the features of LTE-A and its requirements fulfilled in release 11 and 12 for LTE-A is described.
\end{abstract}

\section{INTRODUCTION}

The increasing demand for mobile broadband services with high data rate, speed and quality of service has been the motivation for the 3GPP to develop LTE technology. The release 8 of LTE allows a bandwidth of $20 \mathrm{MHz}$ with a peak data rate of $300 \mathrm{Mb} / \mathrm{s}$ on the downlink and $75 \mathrm{Mb} / \mathrm{s}$ on the uplink whereas release 9 introduced features of multimedia broadcast services, location based services, and other enhancements like dual layer beamforming, commercial mobile alerts from release 8 . However, the advanced version of LTE meets the requirements imposed by IMT (International Mobile Telecommunication) to Fourth Generation. With a peak data rate of $1 \mathrm{Gbps}$ with operating bandwidth up to $100 \mathrm{MHz}$ for the downlink, it provides low latency as compared to LTE,[20]. Its efficient interference management and reduced operational costs make LTE-A popular among operators. Its overall capacity, network management, quality of service management are the attributes that make LTE-A to give the best performance. Its further releases 11 and 12 provide further enhancements in its MIMO, carrier aggregation, and spectrum efficiency to boost speed and solve data traffic issues which will be further discussed,[19]. We have also given an overview on the technological improvements done in release 11 and 12 .

\section{LTE Advanced Features}

\section{A. LTE carrier aggregation enhancements}

Carrier aggregation (CA) is one of the main features of LTE-A as it meets the peak data rate requirements of $1 \mathrm{GBps}$ and $500 \mathrm{Mbps}$ in the uplink as well as downlink. It supports both Frequency division Duplex (FDD) and Time Division Duplex (TDD). CA is designed to support backward compatibility i.e. LTE devices of release 8 and 9 must be able to support devices of release 10,[4]. In CA, data is transmitted through multiple LTE carriers called Component Carriers (CC) in contiguous or non-contiguous manner. CA enables extension of bandwidth and aims in achieving spectral efficiency. A maximum bandwidth of $100 \mathrm{MHz}$ can be achieved by aggregating 5 carriers each of $20 \mathrm{MHz}$ as shown below:

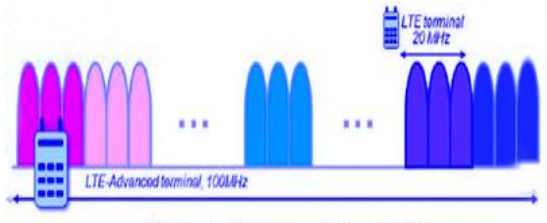

(b) Non-contiguous carrier aggregation

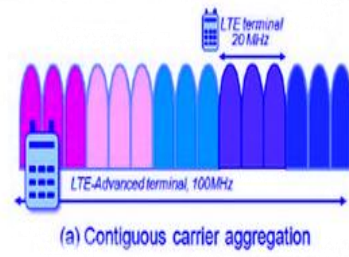

Fig. 1. Carrier aggregation
The component carrier can have a bandwidth of $1.4,3,5,10$, 15 or $20 \mathrm{MHz}$ and a maximum of five component carriers can be aggregated,[20]. Hence the maximum bandwidth is 100 $\mathrm{MHz}$ In CA, broadband transmission is enabled through the communication of multiple CCs exceeding $20 \mathrm{MHz}$ of bandwidth. There are two types of CAs:

a) Contiguous inter-band carrier aggregation

b) Contiguous intra-band carrier aggregation and Noncontiguous intra-band carrier aggregation

In contiguous inter-band $\mathrm{CA}$, the frequency arrangement is such that communication between CCs is achieved by a contiguous band greater than $20 \mathrm{MHz}$. In non-contiguous intraband carrier aggregation, the communication is achieved by the use of two different carrier frequency bands. The use of two frequency bands helps in achieving higher throughput and improves stability of carriers with different propagation environments. In non-contiguous intra-band CA, communication is achieved by using multiple carriers in the same frequency band.This type of CA is observed among multiple operators where network is shared among them. The CA usage scenarios include aggregation of multiple contiguous CCs within the same band, aggregation of multiple non-contiguous CCs within the same band and aggregation of

- Sravanthi Kanchi, Shubhrika Sandilya, Deesha Bhosale, Adwait Pitkar

- Vidyalankar Institute of Technology, Wadala, Mumbai.

sravanthi.kanchi@vit.edu.in,

shubhrika.sandilya@vit.edu.in, deesha.bhosale@vit.edu.in, adwait.pitkar@vit.edu.in

- Mayur Gondhalekar: Former Assistant Professor, Vidyalankar Institute of Technology, Wadala, Mumbai, India, gondhalekar.mayur@gmail.com 
multiple non-contiguous CCs located in different bands. Using a proper architecture is a must to implement any one of the usage scenarios,[17].

Physical Layer: In the downlink of the physical layer, LTE-A has the same OFDMA based radio accessed system as in release.8,[20],[21] .When bandwidth is extended using CA, the synchronization signal carries out cell search which is transmitted on the center frequency of each cell where each cell has an arranged center frequency of $100 \mathrm{kHz}$ raster. Physical Broadband channel (PBCH) is also multiplexed in the same way. Thus, $\mathrm{PBCH}$ and SS can be used in all CCs. Adaptive modulation and coding (AMC) and Hybrid Automatic Repeat Request(HARQ) is performed separately on each CC , in units of CCs known as transport block and these blocks are further combined together to form a single CC. This is performed on the Physical Downlink Shared Channel (PDSCH). These transport blocks can be reused as mentioned according to specifications in release 8 . In the uplink, the signals are generated by the SC-FDMA system.

There are three new control downlink control channels: the Physical Control Format Indicator Channel (PCFICH), the Physical Downlink Control Channel (PDCCH) and the Physical Hybrid ARQ Indicator Channel (PHICH),[17].

PCFICH: in this channel, each CC will have its own information on the size of the control region.

PDCCH: This channel performs resource allocation in two ways. The first way is done resource manager where it allocates resources to PDSCH and $\mathrm{PUSCH}$ in the same CC,[17]. The other way of resource allocation is done by Carrier Indicator Field (CIF). Using CIF resources from one single CC can be allocated into multiple CCs. This allows high scheduling flexibility and reduces interference among CCs.

PHICH: This channel includes the same aspects of transmission as present in release 8 . This channel is used in the CC only when scheduling information is to be transmitted on the uplink.

\section{B. MIMO, Multiple Input Multiple Output - or spatial multiplexing}

Due to increasing mobile traffic over the years, the requirement of advanced MIMO channel transmission and higher peak efficiency than LTE has been gaining wide importance. MIMO is used to increase the overall bitrate through transmission of two (or more) different data streams on two (or more) different antennas - using the same resources in both frequency and time, separated only through use of different reference signals - to be received by two or more antennas,[7]. LTE advanced supports the configuration of 8 antennas in the downlink and 4 antennas in the uplink. Multiple antenna techniques play an important role in increasing spectral efficiency, average cell throughput and cell edge performance.

\section{Layered OFDMA}

The technique used for radio transmission and reception is OFDMA (orthogonal frequency division multiple access. OFDMA dynamically maps traffic based on their instantaneous throughput. It has its own layered control signalling structure, layered environment for both down-link and up-link and layered transmission bandwidth. The support of layered environment helps in achieving high data rate (high throughput) or widest coverage according to respective radio environments such as macro, micro, indoor, and hotspot cells,[4]. It involves key radio access technologies such as fast inter cell radio management, multi-antenna transmission and widest coverage area to achieve high level capacity and cell-edge spectrum efficiency.

\section{Coordinated multi point transmission and reception(COMP)}

Coordinated multipoint transmission and reception involves transmission/reception of signals to/from user equipments (UEs) located at multiple cell sites. CoMP is applied in the downlink by performing a coordinated transmission from the base station. Whereas interference in the uplink can be reduced by means of a coordinated reception in eNBs. It provides enhanced reception performance thus increases received power improving end user service quality. COMP technology prevents base station interference and abrupt call disconnections in coverage boundary area. It has increased throughput and has decreased interference on cell edge, thus turn all interference signals on cell borders into useful signal that improve the system,[6],[7]. Currently COMP schemes are evaluated under various scenarios with remote radio head $(\mathrm{RRH})$ which ensures high capacity and low latency backhaul.

\section{E. Relay technology}

For efficient planning of heterogeneous network, 3GPP LTE advanced concept of relay nodes. The relay node establish wireless connection with radio access network via a donor cell. The relay nodes are low power base station that provide enhanced coverage in targeted areas in low costs without the need for a wired backhaul connection,[8] .In addition wireless relays can increase throughput, provide group mobility and capacity at cell edges. Layer 3 relays have been standardized by 3 GPP release with LTE-A in which they have a unique physical cell ID to ensure UE knows it is connected to a relay node and treats relay node as base station layer. The principle of operation involves demodulating and decoding the incoming signal and re-modulating and re-encoding the signal before amplified version is transmitted.

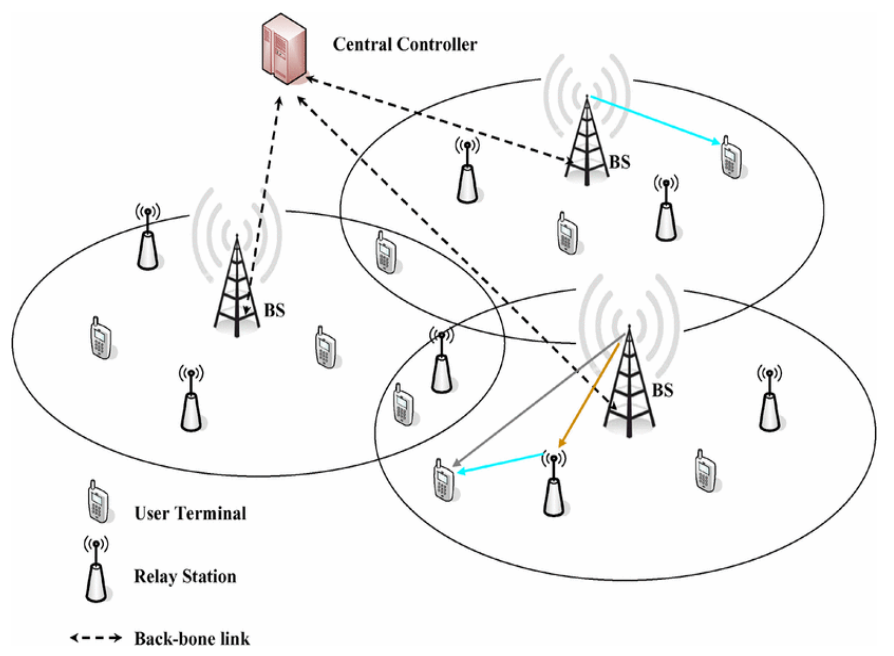

Fig 2. Relay technology 
TABLE I

LAYERS IN RELAY NODES

\begin{tabular}{|c|c|c|}
\hline$\frac{\text { Layer }}{\frac{1}{\text { relay }}}$ & $\begin{array}{l}\text { ADVANTAGES: } \\
\text { 1)Simple and in } \\
\text { expansive. } \\
\text { 2)minimal } \\
\text { impact } \\
\text { standard on } \\
\text { specifications. } \\
\text { DISADVANTAG } \\
\text { E } \\
\text { 1)noise is } \\
\text { amplified with } \\
\text { the signal }\end{array}$ & 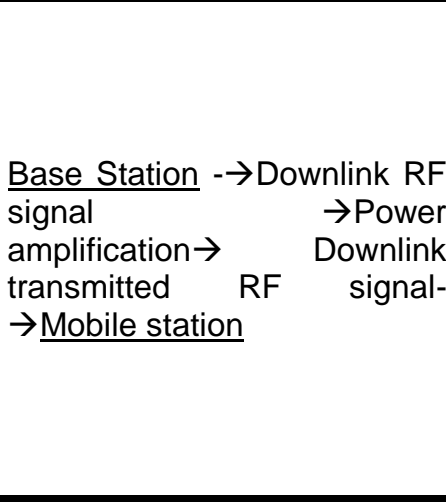 \\
\hline$\frac{\text { Layer }}{\frac{2}{\text { relay }}}$ & $\begin{array}{l}\text { ADVANTAGES } \\
\text { 1)elimination of } \\
\text { noise. } \\
\text { DISADVANTAG } \\
\frac{\text { ES }}{1 \text { )process delay }} \\
\text { due to } \\
\text { modulation tdemodulation } \\
\text { /de }\end{array}$ & $\begin{array}{l}\text { Base Station } \\
\rightarrow \text { Demodulation } \\
\rightarrow \text { Encoding/modulation-- } \\
\rightarrow \text { Power amplification- } \\
\rightarrow \text { Mobile station. }\end{array}$ \\
\hline $\begin{array}{l}\text { Layer } \\
3 \\
\text { relay }\end{array}$ & $\begin{array}{l}\frac{\text { ADVANTAGES }}{\text { 1)elimination of }} \\
\text { noise } \\
\text { 2)small impact } \\
\text { of standard } \\
\text { specification. } \\
\text { DISADVANTAG } \\
\frac{\text { ES }}{\text { 1)layer3 }} \\
\text { process } \\
\text { delay(user data } \\
\text { regeneration } \\
\text { processing }\end{array}$ & $\begin{array}{l}\text { Base Station }-\rightarrow \text { user data } \\
\text { regeneration } \rightarrow \text { Demodulation } \\
-\rightarrow \text { user data transmission } \\
\text { processing } \rightarrow \text { Encoding/modul } \\
\text { ation-- } \rightarrow \text { Power amplification- } \\
\rightarrow \text { Mobile station. }\end{array}$ \\
\hline
\end{tabular}

\section{F. Heterogeneous networks}

Using a mix of macro, pico, femto and relay base-stations, heterogeneous networks enable flexible and low-cost deployments and provide a uniform broadband experience to users anywhere in the network,[6]. Heterogeneous networks, utilizing a diverse set of base stations, can be deployed to improve spectral efficiency per unit area,[6]. The low-power base stations can be deployed to eliminate coverage holes in the macro-only system and improve capacity in hot spots,[7].

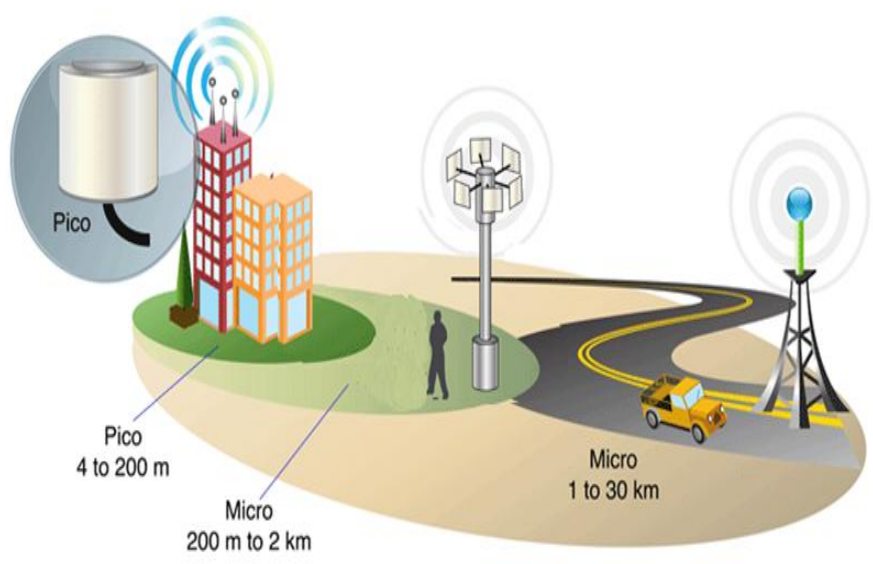

Fig. 3. Heterogeneous Networks

In a homogeneous network, each mobile terminal is served by the base stations with the strongest signal strength, while the unwanted signals received from other base stations are usually treated as interference. In such systems, smarter resource coordination among base stations, better server selection strategies and more advanced techniques for efficient interference management can provide substantial gains in throughput and user experience as compared to a conventional approach of deploying cellular network infrastructure.

\section{Enhancements In LTE-A}

\section{A. Release 11}

Enhanced PDCCH: Release 11 has introduced a new downlink control channel (EPDCCH) Enhanced Physical Downlink Channel. It is an important feature as it supports CoMP, downlink MIMO as well as beamforming and frequency domain (ICIC),[2]. It operates on a new carrier type. One of its major goal is to achieve an improved spatial reuse of control channel resources. It coexists on the same carrier type as that of release 8 and release 10 devices. Enhanced $\mathrm{PDCCH}$ has a similar design as to the PDSCH in release 8. It uses resources same as that of PDSCH.

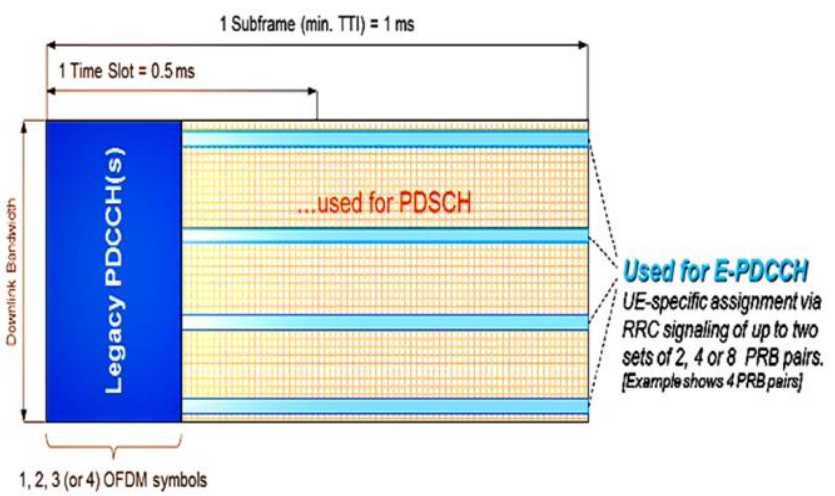

Fig. 4. $\mathrm{EPDCCH}$

An RRC signal will decide which sub-frames have to monitor EPDCCH when indicated to the device. The signal can also 
decide UE to monitor Resource Block or RB pairs. Resource Block pairs carry the EPDCCH. These RB pairs could be of size 2, 4 or 8. Each RB pair consists of a number of Enhanced Control Channel Elements (ECCE). Each ECCE consists of 4 or 8 resource element groups or EREG. EPDCCH uses one or more ECCE for its transmission. EREG is further organized into EREG groups. Group \#0 represents the EREG group formed with indices $0,4,8$ and 12 . There are 13 such groups in all.

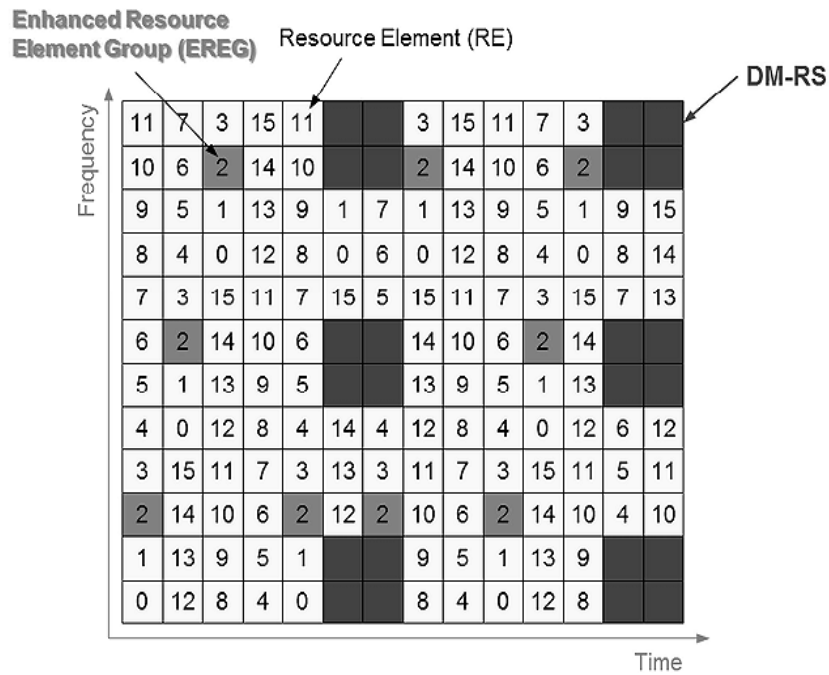

Fig. 5.Group \#0 EREG group

EPDCCH uses two types of transmission i.e. localized transmission and distributed transmission. If an EREG group is located in a single RB pair, the transmission is localized. It allows frequency -selective scheduling, using favourable subbands based on radio channel feedback gained by the device. If the feedback obtained is not proper, then EPDCCH uses distributed transmission mode for transmission.

1) $C A$ enhancement in release 11: In release 11, the $C A$ feature has been further enhanced using multiple timing advances. A TAG (Timing Advance Group) was introduced in order to implement multiple timing advances in CA,[2]. The TAG containing a PCell is called Primary Timing Advance Group whereas the TAG containing SCell is called as Secondary Timing Advance Group. As CA is limited to maximum of only two downlink carriers from RF (3GPP RAN4) perspective, only two TAGs are allowed. Just as the initial timing advance procedure in release 8,[4], the initial uplink UL timing alignment is obtained with STAG using eNB random access procedure in release 11. With $\mathrm{RACH}$ resources an SCell can be configured with an sTAG. Further, eNodeB initiates the RACH transmission on the secondary cells by the $\mathrm{PDCCH}$ order sent on the primary cell. Then, the SCell response is sent to a PCell using RA-RNTI as in release 8 . The downlink frame of the SCell is tracked by the UE. It adjusts the UL timing which follows the timing commands of eNB. A modification has been done in the MAC command control element in order to bring timing advances commands into implementation successfully,[2]. The control element consists of a new 2 bit TAG id and a 6 bit timing advance command field. Thus, multiple timing advances in CA ensure that transmissions are faster and they impact the physical layer further.

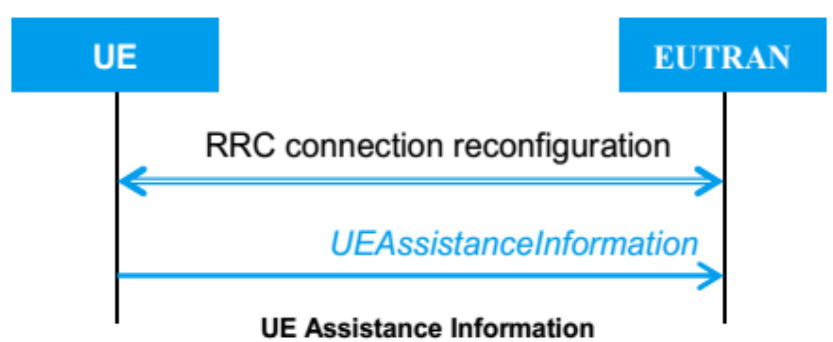

Fig. 6.UE Assistance Information

2) CoMP enhancement in release 11: There has been an enhancement in release 11 in the downlink and the uplink of CoMP,[2]. In the downlink, Joint Transmission is carried out which allows data transmission simultaneously from multiple points to single or multiple UE. In Joint transmission, data transmission takes place between multiple points within each sub-frame. Because of JT, the data at the UE is available at multiple points. The aim of downlink CoMP, is to improve signal quality at the receiver as well as the average throughput. Beam-forming or Co-ordinated scheduling is performed for a single transmission point. The main aim of Beam-forming is to reduce interference between cells thereby increasing the system's performance. Joint reception is performed on the uplink of CoMP,[2]. UE transmits the PUSCH which is jointly received at multiple points. In beam-forming the $\mathrm{PUSCH}$ can be obtained at one point only .Release 11 introduces the concept of Virtual Cell Identities (VCID). It helps in avoiding high interference in scenarios where a macro cell provides coverage and several small cells are used for capacity. This type of situation occurs when macro cell and small cells have the same identity. Due to VCID, transmission point and reception point do not appear the same anymore. Therefore, they are used in the uplink of CoMP.

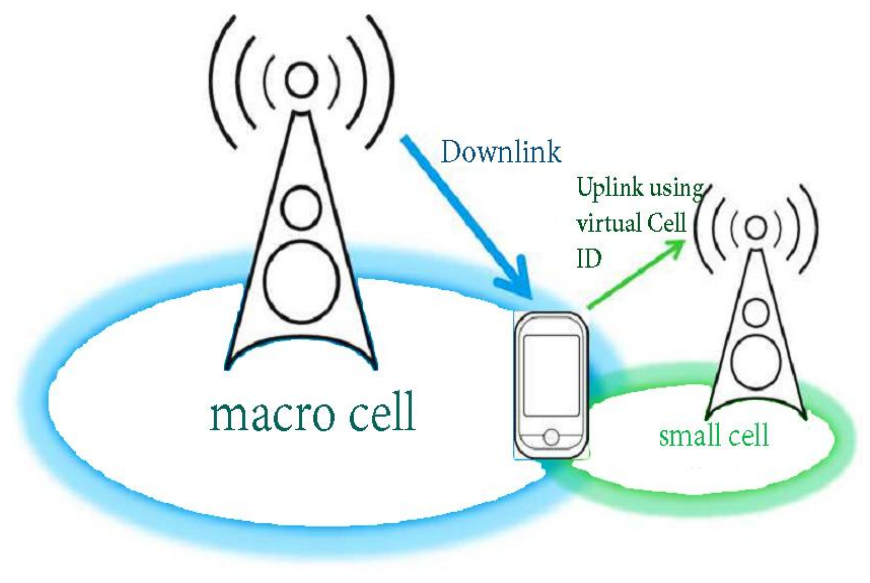

Fig. 7. CoMP enhancement scenario

\section{B. Release 12}

As the number of connecting devices are increasing, there will be huge load on the network. Also the access for data will be increasing due to which there will be a massive traffic data volume. There would be a need for applications with varying requirements and characteristics .Future wireless systems must be able to offer mobile broadband services at multi Gbps data rates to users locally. To satisfy all these, the LTE radio 
access technology (RAT) has made the following enhancements:

1. Enhancement in multi-antenna transmissions and advanced receivers in order to mitigate inter-cell and intracell interference thus improving the system's performance.

2. Improved network efficiency by eliminating unnecessary transmission activity thereby reducing interference and leading to improved data rates in heterogeneous deployments.

3. By integrating WIFI with LTE at local area level provides overall improved mobile broadband performance and better overall radio resource management. Thus, allowing operators to give better user experience for its users as well as boosting the capacity to handle data traffic.

4. USE OF NEW USE-CASES:

1)Machine type Communication (MTC): 3GPP has made enhancements in this area with the aim to reduce energy consumption of MTC and reduce costs,[19]. This is done by introducing low cost MTC devices due to which there would be extended coverage options in challenging locations for MTC services and by handling large number of devices per cell.

2)Discovery and Communication: LTE uses D2D (Device to Device) communication. It involves proximity detection in which a device carries out a search in its surroundings and detects the presence of another device. And further performs the step of examining the possibility of D2D communication.

\section{Conclusions}

LTE-Advanced standardization in the 3GPP specification Release 10 was designed to meet $4 \mathrm{G}$ requirements as IMT. After that, there have been many enhancements and there has been a significant technological progress in improving the requirements of LTE-A as specified in its releases 11 and 12 . Along with the enhancements in the general features of LTE release 10 , release 11 has functionalities to reduce inter cell interference and efficiently supports heterogeneous network topologies. Release 12 further enhances release 11 by improving the efficiency of the network and has helped in overcoming the minor limitations in data traffic in its previous releases. Due to this, LTE-A has been able to give the best user experience. The network has proven to support and handle multiple devices at a single time. It has been capable to address 1000x in mobile data. LTE advanced is the next milestone in the evolution of LTE. It has its main goal for providing high data rate, flexibility in spectrum, efficient signal strength and quality network management. It is one of the fastest upcoming technologies which will change the future of wireless communication.

\section{REFERENCES}

[1]. 4G Mobile Broadband Evolution: 3GPP Release 10 and Be-yond HSPA+, SAE/LTE and LTE-advanced, 4G Americas, January 2012, pp. 80-95. Available on: http://www.4gamericas.org/documents/4G\%20Americ as 3GPP Rel-10 Beyond 2.1.11\%20.pdf

[2]. Rohde \& Schwarz; LTE Advanced 3GPP (REI. 11) Technology pp.16-20 Available on http://www.rohdeschwarz.com/en/applications/lte-advanced-3gpp- rel.11-technology-introduction-applicationnote 56280- 42753.htmly Introduction White Paper.pdf

[3]. Hyung G Myung; Technical Overview of 3GPP LTE ,May2008,pg.37 Available on: http://www.docstoc.com/docs/159510677/TechnicalOverview-of-3GPP-LTEDocStoc

[4]. Edward Kasem, Jan Prokopec: The evolution of LTE to LTE-Advanced and the corresponding changes in the uplink reference signals Volume. 3, No. 2, June 2012,pp. $1-5$ Available on http://www.elektrorevue.cz/en/download/theevolution-of-Ite-to-Ite-advanced-and-thecorresponding-changes-in-the-uplink-referencesignals.pdf

[5]. 4G Mobile Broadband Evolution- Rel 10 Rel 11 Beyond October 2012 pp.57-61 Available on; http://www.4gamericas.org/documents/4G\%20Mobile \%20Broadband\%20EvolutionRel/2010/Rel/2011/Beyond October/202012/20PPT.pdf

[6]. Motorola long term evolution LTE a technical overview: technical white paper 2007,pp.3 Available on:

http://www.motorolasolutions.com/web/Business/Solut ions/Industry/Solutions/Service/Providers/WirelessOp erators/LTE/ Document/Static/Files/6834 MotDoc N ew.pdf

[7]. Tata Consultancy Services LTE-Advanced: Future of Mobile Broadband Sep 2009 pp.5-9 Available on http://www.tcs.com/SiteCollectionDocuments/WhitePa pers/EIS Whitepaper LTE Advanced Future of Mo bile broadband 09 2009.pdf

[8]. LTE-Advanced Next-Generation Wireless Broadband Technology: IEEE Wireless Communications June $2010 \quad$ Available on: http://ltewatch.blogspot.com/2012/11/ltewatchtechnical-papers-on-lte.html

[9]. Jeanette Wannstrom: LTE-Advanced for 3GPP,May 2012 pp:1-4 Available on: http://www.3gpp.org/Keywords-Acronyms.pdf

[10]. Ian F. Akyildiz , David M. Gutierrez-Estevez, Elias Chavarria Reyes: The evolution to 4G cellular systems: LTE-Advanced, Physical Communication 3,pp.4,10,21 Available on: http://www.journals.elsevier.com/physicalcommunication

[11]. Eiko Seidel:Nomor Researh GmbH Progress on LTE Advanced - the new 4G standard July 2008 pp.2-5 Available on- http://www.nomor.de/

[12]. Nokia Siemens Networks LTE-Advanced white paper: The advanced LTE toolbox for more efficient delivery of better user experience ,pp.5-15 Available on- 
http://nsn.com/system/files/document/lteadvanced technical whitepaper 22032011 v03 lowres.pdf

[13]. LTE Advanced: The 4G Mobile Broadband Technology, International Journal Of Computer Applications (0975 - 8887) Volume 13- No.5, January 2011 pp.2-4 Available onhttp://www.ijcta.com/documents/volumes/vol2issue4/ij cta2011020448.pdf

[14]. Stefan Schwarz, Josep Colom Ikuno, Michal Imko, Martin Taranetz, Qi Wang, And Markus Rupp: Pushing the Limits of LTE: A Survey on Research Enhancing the Standard ,IEEE May 2013 Available on- http://www.ieeeexplore.com

[15]. Relay technology in LTE Advanced:technical journal $\begin{array}{lllll}\text { Vol } & 12 & \text { No.2 } & \text { Available on }\end{array}$ http://www.nttdocomo.co.jp/english/binary/pdf/corpora te/technology/rd/technical journal/bn/vol12 2/vol12 2 029en.pdf

[16]. Christopher Cox, "An Introduction to LTE", LTE,LTEAdvanced, SAE, 4G mobile communications, $1^{\text {st }}$ ed, John Wiley and Sons Ltd. ch 18 , ch 19 , ch 20.

[17]. Guillaume de la Roche, Andres Alayon Glazunov, Ben Allen, "LTE-Advanced and Next Generation Wireless Networks Channel Modeling and Propagation “John Wiley and Sons Ltd. 2013 ,ch 1, ch 2 ,ch 15.

[18]. Novel Mobile Radio(Nomor) Research: 3GPP LTE-A Standardization in in Release 12 And Beyond, January 2013, pg.1-2 Available on http://www.nomor.de/uploads/fd/24/fd24709a64bc490 a083a8eba6d3cc2cb/NoMoR LTEA Rel12 and Beyond 2013-01.pdf

[19]. Erricson White Paper : LTE- Release 12 - Taking Another Step Toward The Networked Society, January 2013,pg. 1-12 Available on http://www.ericsson.com/res/docs/whitepapers/wp-lterelease-12.pdf

[20]. Nobuhiko Miki, Mikio Iwamura, Yoshihisa Kisimara,Umesh Anil, Hiroyuki Ishi, Technology Reports : Special articles on LTE-Advanced Technology-On Going Evolution of LTE toward IMTAdvanced - $\quad$ CA Available on http://www.nttdocomo.co.jp/english/binary/pdf/corpora te/technology/rd/technical journal/bn/vol12 2/vol12 2 010en.pdf

[21]. Klaus Ingeman Pederson, Luis Guilherme Uzeda, Hung Nguyen, Yuanye Wang, Frank Frederiksen, Claudio Rosa, Carrier Aggregation for LTE-Advanced : Functionality And Performance Aspects , pg.1-7, Available on http://vbn.aau.dk/files/57467303/CA paper V17.pdf 